\title{
Current Trends in the Diagnosis of Primary Ciliary Dyskinesia
}

\author{
Eric P. Wartchow
}

Department of Pathology, Children's Hospital Colorado, Aurora, CO 80045

Primary ciliary dyskinesia (PCD) is a rare genetic disease caused by abnormal cilia function within the respiratory airways [1]. Characterized by a reduced or total lack of clearance of secreted mucous, the associated chronic infection and inflammation may result in severe, irreversible respiratory damage. The diagnostic process may be quite difficult and should evaluate the patient's cilia function and structure through a multimodal approach that relies on a careful review of the clinical history and the use of appropriate laboratory tests. Currently, the definitive diagnostic test is the identification of a consistent ultrastructural abnormality within the cilia axoneme by electron microscopy (EM) with additional support oftentimes provided by other ancillary techniques.

Diagnostic Considerations

\section{Clinical Symptoms}

Although many of the disease symptoms are present at birth, diagnosis is often delayed until later in childhood or even adulthood because disease symptoms mimic other more common respiratory diseases and because of a lack of an ideal "gold standard" reference test [2]. The classic clinical symptoms of PCD include a history of neonatal respiratory syndrome, chronic upper and lower airway disease, recurrent otitis media, and bronchiectasis [3]. Situs inversus is observed in approximately one half of all PCD patients.

\section{Nasal Nitric Oxide Testing}

Patients with PCD produce lower levels of exhaled nasal nitric oxide (nNO) [4]. Although the exact nature of the relationship between $\mathrm{nNO}$ and PCD has yet to be determined, the use of nNO testing has emerged as a powerful screening mechanism in patients $>5$ years old. However, given that other conditions with similar, overlapping symptoms also report low levels of $\mathrm{nNO}$, this test cannot independently confirm a diagnosis of PCD. Likewise, normal nNO levels cannot definitively exclude PCD [5].

\section{Motility Studies}

While direct, in situ observation of respiratory epithelial cell cilia has been shown to result in unacceptable levels of false negative reports, the quantitative analysis of cilia beat patterns and beat frequency using high speed video recordings of cilia movement has been reported to be an effective screening technique [6,7]. Proponents of this screening technique recommend that if normal ciliary beat patterns and frequency are observed, PCD can be excluded and further PCD diagnostic tests can be aborted [8].

\section{Genetic Testing}

Researchers have been trying to elucidate the genes that may harbor genetic mutations causing PCD, and to date have identified mutations within 11 genes on eight different chromosomes. Currently, known mutations are believed to account for less than $60 \%$ of all cases of PCD [9]. Perhaps the most useful of the recently identified mutations is that of DNAH11, which has been confirmed in patients with clinical manifestations of PCD, low levels of nasal NO, but with normal cilia ultrastructure by EM [10]. Testing 
for this and other known mutations causing PCD is commercially available, however the gene sequencing panel is quite expensive and currently not covered by medical insurance providers.

\section{Transmission Electron Microscopy}

The use of transmission electron microscopy to identify consistent structural defects of the ciliary axoneme remains the most common, and often the only technique used for establishing a diagnosis of PCD. EM evaluation involves observation of cilia in cross-section to determine the presence of the structural and motility-producing components. Such components include the normal 9+2 microtubule architecture, the presence of the inner and outer dynein arm motor proteins, and the proper formation of the radial spoke/central pair apparatus. While the technique has some limitations (specimen collection and preparation, interpretation of images, requirement of specialized equipment), electron microscopy, working in concert with other screening techniques and genetic testing of known mutations, can be expected to remain the gold standard diagnostic technique for the next years, or even decades, to come.

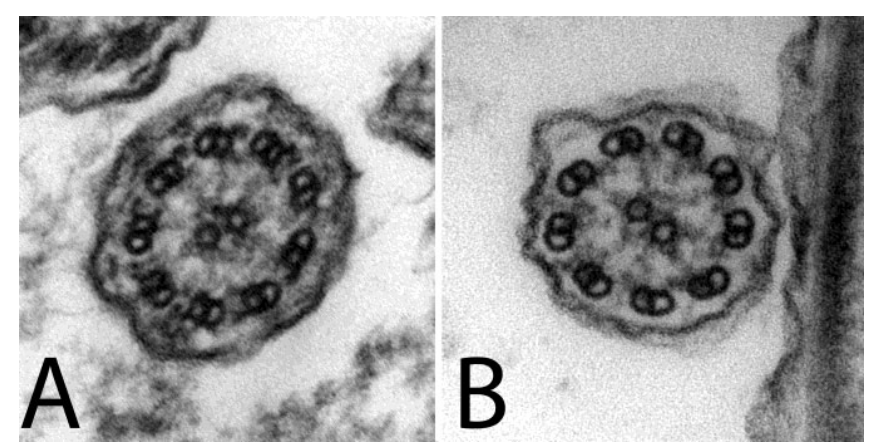

Figure 1: Cilia ultrastructure observed by transmission electron microscopy. (A) Normal cilium crosssection demonstrating the presence of all axonemal components including 9+2 microtubular arrangement, inner and outer dynein arms, and radial spokes/central apparatus. (B) Abnormal cilium architecture demonstrating missing outer dynein arms.

References:

[1] B. Afzelius, Science 193 (1976), p. 317-319.

[2] P. Stillwell, E Wartchow, S Sagel, Pediatr Allergy Immunol Pulmonol 24 (2011), p. 191-196.

[3] P Noone, et al, Am J Respir Crit Care Med 169 (2004), p. 459-467.

[4] B Karadag, et al., Eur Respir J 13 (1999), p. 1402-1405.

[5] S Sagel, Journal of Pediatrics 159 (2011), p. 363-365.

[6] J Carson, Laryngoscope 121 (2011), p. 2047.

[7] M Chilvers, et al., J Allergy Clin Immunol 112 (2003), p. 518-524.

[8] C Hogg, A Bush, Thorax 67 (2012), p. 377-378.

[9] Ambry Genetics (http://www.ambrygen.com).

[10] M Knowles et al., Thorax 67 (2012), p. 433-441. 